\title{
ON THE COMPLEXITY OF TOPOLOGICAL CONJUGACY OF TOEPLITZ SUBSHIFTS
}

\author{
MARCIN SABOK AND TODOR TSANKOV
}

\begin{abstract}
In this paper, we study the descriptive set theoretic complexity of the equivalence relation of conjugacy of Toeplitz subshifts of a residually finite group $G$. On the one hand, we show that if $G=\mathbb{Z}$, then topological conjugacy on Toeplitz subshifts with separated holes is amenable. In contrast, if $G$ is non-amenable, then conjugacy of Toeplitz $G$-subshifts is a non-amenable equivalence relation. The results were motivated by a general question, asked by Gao, Jackson and Seward, about the complexity of conjugacy for minimal, free subshifts of countable groups.
\end{abstract}

\section{INTRODUCTION}

The theory of definable equivalence relations offers tools for classifying the complexity of equivalence relations arising from various isomorphism problems. An important class of Borel equivalence relations is given by the countable ones, i.e., those with countable classes. It is a classical result of Feldman and Moore that every such equivalence relation arises as the orbit equivalence relation of a Borel action of a countable group; and thus, from the beginning, the theory is intimately connected to that of dynamical systems from where it has borrowed most of its tools and techniques. The descriptive set theoretic approach to those equivalence relations has been developed over the last twenty years by Dougherty, Jackson, Kechris, Louveau, Hjorth, Thomas and others (see, e.g., [13, 5, 12]).

The natural comparison of equivalence relation is given by Borel reducibility and the simplest ones are those which are smooth, i.e., admit real numbers as complete invariants (or, equivalently, admit a Borel transversal). The next level of the complexity hierarchy is formed by the hyperfinite ones, equivalently, those induced by Borel actions of the group of integers $\mathbb{Z}$. There is also a universal countable Borel equivalence relation, which is maximal in the quasi-order of Borel reducibility, an example is the orbit equivalence relation $F_{2} \curvearrowright 2^{F_{2}}$.

In topological dynamics, one of the most commonly considered types of dynamical systems are the subshifts (also known as Bernoulli subflows or symbolic dynamical systems). For a countable group $G$, the Bernoulli shift is the action of $G$ on $2^{G}$ defined by the formula: $(g \cdot x)(h)=x\left(g^{-1} h\right)$ for all $g, h \in G, x \in 2^{G}$. A $G$-subshift is a closed nonempty subset $S \subseteq 2^{G}$ which is invariant under the action of $G$. The natural isomorphism relation of subshifts is topological conjugacy: two subshifts $S$ and $T$ are conjugate if there is a homeomorphism $f: S \rightarrow T$ which commutes with the action of $G$. This is the equivalence relation that we study in this paper. Often, special classes of subshifts are of interest in dynamics. A subshift $S$ is minimal if it does not contain any proper subshift, or equivalently, if every orbit is dense. It is free if for any $x \in S$ and any $g \in G$ different from $1_{G}$, we have $g \cdot x \neq x$.

The first author was partially supported by NSERC and the Polish Ministry of Science and Higher Education (MNiSW) through the grant Mobilność Plus. The second author was partially supported by the ANR contracts GrupoLoco (ANR-11-JS01-008) and GAMME (ANR-14-CE250004). 
It is worth noting that while many countable equivalence relations arise naturally from group actions, some do not, and their study is usually more difficult because most of the available tools are dynamical in nature and require the presence of a (natural) group action. Some notable examples where there is no natural group action that gives the equivalence relation are Turing equivalence, isomorphism of (various classes of) finitely generated groups, and topological conjugacy of subshifts. (However, for subshifts, there is still a group action present that can be exploited: see Section 4, )

The recent monograph of Gao, Jackson, and Seward [9] studies the complexity of topological conjugacy of free, minimal subshifts. (In fact, before their construction, it was an open problem whether such subshifts necessarily exist for every countable $G$.) It follows essentially from a classical result of Curtis, Hedlund and Lyndon (see [19] or [9. Lemma 9.2.1]) that for any countable group $G$, topological conjugacy of $G$-subshifts is a countable Borel equivalence relation. Gao, Jackson and Seward showed [9, Corollary 1.5.4] that if $G$ is infinite, this equivalence relation is not smooth, and that if $G$ is locally finite, then it is hyperfinite [9, Theorem 1.5.6]. They also pose the general question [9, Problem 9.4.11] to determine the complexity of this equivalence relation for an arbitrary countable group $G$. This was an important motivating question for our work and in Theorem 1.2, we provide a partial answer.

Clemens [1] proved that the topological conjugacy of $\mathbb{Z}$-subshifts is a universal countable Borel equivalence relation. However, his construction produces subshifts that are far from minimal and it remains an open question whether isomorphism of minimal $\mathbb{Z}$-subshifts is universal. This question is connected with the conjecture of Thomas [23, Conjecture 1.2] that isomorphism of finitely generated, amenable, simple groups is universal. It follows from the results of Matui [21, Giordano, Putnam, Skau [10] and a recent result of Juschenko and Monod [14] that the computation of the topological full group of a minimal $\mathbb{Z}$-subshift provides a reduction from the (flip-) conjugacy of minimal $\mathbb{Z}$-subshifts to isomorphism of finitely generated, simple, amenable groups. Another related result was recently proved by Williams 24, who showed that isomorphism of finitely generated, solvable groups is weakly universal.

The focus of this paper is studying the complexity of the conjugacy equivalence relation for the class of Toeplitz subshifts of residually finite groups. This class of subshifts is well-known and appears in many contexts. For example, Downarowicz [7] showed that that any Choquet simplex can be realized as the simplex of invariant measures of a Toeplitz subshift. (This result was recently generalized to arbitrary amenable, residually finite groups by Cortez and Petite 4.) An important feature of Toeplitz words is that they can be constructed in stages, which allows a fair amount of control. We briefly recall the classical definition for $G=\mathbb{Z}$ here and postpone the general one for residually finite groups to Section 2.

A word $x \in 2^{\mathbb{Z}}$ is Toeplitz if every symbol occurs periodically, i.e., for every $n \in \mathbb{N}$, there exists $k$ such that $x(n+k i)=x(n)$ for all $i \in \mathbb{Z}$. A subshift $S \subseteq 2^{\mathbb{Z}}$ is Toeplitz if it is equal to the closure of the orbit of some Toeplitz word. It is easy to check that every Toeplitz subshift is minimal.

The topological conjugacy relation for Toeplitz $\mathbb{Z}$-subshifts has been studied by Downarowicz, Kwiatkowski and Lacroix [6] and it essentially follows from their results that topological conjugacy of pointed Toeplitz flows (i.e., the relation $E$ on Toeplitz words, such that $x E y$ if there is a an $\mathbb{Z}$-equivariant homeomorphism from $\overline{\mathbb{Z} \cdot x}$ to $\overline{\mathbb{Z} \cdot y}$ that maps $x$ to $y$ ) is a hyperfinite equivalence relation. The latter seems to indicate that the topological conjugacy relation for Toeplitz $\mathbb{Z}$-subshifts should not be too complicated. However, we have only been able to treat the case 
of Toeplitz subshifts with separated holes, a special but important class. The first of our main results is the following.

Theorem 1.1. For $G=\mathbb{Z}$, the equivalence relation of conjugacy on Toeplitz subshifts with separated holes is amenable and therefore hyperfinite $\mu$-a.e. for every Borel probability measure $\mu$ on the set of subshifts.

We postpone the definitions to Section 3 and just recall that an amenable equivalence relation is hyperfinite a.e. with respect to any probability measure but it is an open problem whether it must be hyperfinite everywhere. We do not know whether the above equivalence relation is hyperfinite.

The second part of the paper deals with Toeplitz subshifts of residually finite, non-amenable groups. For those, we prove that topological conjugacy is somewhat complicated.

Theorem 1.2. If $G$ is a non-amenable, residually finite group, then the equivalence relation of conjugacy on the set of free, Toeplitz G-subshifts is not hyperfinite.

The proof of this theorem proceeds by constructing a probability measure $\mu$ on the set of Toeplitz subshifts which is invariant under a suitable action of the group $G$, included in the equivalence relation; then we show that the stabilizers of points are amenable and conclude that the equivalence relation is not $\mu$-amenable and thus not hyperfinite.

In the last section of the paper, reinterpreting a result of Downarowicz, Kwiatkowski, and Lacroix, we indicate how topological conjugacy of Toeplitz $\mathbb{Z}$-subshifts is naturally generated by an action of a groupoid which is "hyperfinite-by-compact." This seems to indicate that the equivalence relation is somewhat simple; however, we are not even able to prove that it is not universal. The following question remains open:

Question 1.3. Is topological conjugacy of Toeplitz $\mathbb{Z}$-subshifts hyperfinite? Is this true for an arbitrary residually finite, amenable group?

Acknowledgements. This work was initiated during the stay of the first author at the University Paris 7. He would like to thank the logic group in Paris for their hospitality. Both authors would like to thank Boban Veličković for many valuable discussions. The first author is also indebted to Tomasz Downarowicz for valuable comments.

\section{Toeplitz SUBShifts}

In this section, we recall the definition and collect some basic properties of Toeplitz subshifts for residually finite groups. We also establish some basic definability properties that will be needed later.

Let $G$ be a countable group. Recall that the profinite topology on $G$ is the one with the basis of neighborhoods at $1_{G}$ consisting of all finite index subgroups (or, equivalently, all finite index normal subgroups). $G$ is called residually finite if $\left\{1_{G}\right\}$ is closed in the profinite topology, or, equivalently, if the profinite topology is Hausdorff. For the rest of the paper, $G$ will always be a residually finite group.

The profinite completion of $G$, denoted by $\widehat{G}$, is the completion of the group uniformity defined by this topology; equivalently $\widehat{G}=\lim G / H$, where the limit is taken over all finite index normal subgroups of $G$. This profinite completion is metrizable if $G$ has only countably many subgroups of finite index (for example, if it is finitely generated); even though it is not strictly necessary for what we are doing (as we can always pass to suitable metrizable quotients), we will sometimes assume this for convenience. 
Recall that the left shift action $G \curvearrowright 2^{G}$ is defined by $(g \cdot x)(h)=x\left(g^{-1} h\right)$. A closed subset $S \subseteq 2^{G}$ is called a subshift if it is invariant under this action.

Definition 2.1 (Krieger [17). A word $x \in 2^{G}$ is called Toeplitz if $x: G \rightarrow 2$ is a continuous map for the profinite topology on $G$ (where $2=\{0,1\}$ is taken to be discrete). A subshift $S \subseteq 2^{G}$ is Toeplitz if there exists a Toeplitz word $x$ such that $S=\overline{G \cdot x}$.

Generalizing a well-known fact for $G=\mathbb{Z}$, Krieger showed that every Toeplitz subshift is minimal (see [17, Corollaire 2.5]).

Let

$$
S(G)=\left\{S \subseteq 2^{G}: S \text { is closed and } G \text {-invariant }\right\}
$$

be the set of all $G$-subshifts. This is naturally a compact space with the Vietoris topology. It is easy to check that the family of minimal subshifts as well as that of free subshifts form Borel subsets of $S(G)$. In what follows, we see that being Toeplitz is also a Borel condition and establish a simple selection lemma that will be used later.

We need the following well-known definability property of Baire category notions for which we have not been able to find a suitable reference. It is a slight generalization of [15, 16.1] with the same proof. If $X$ is a Polish space, $F(X)$ denotes the Effros Borel space of closed subsets of $X$. Recall that $\exists^{*} x \in F$ means "for non-meagerly many $x$ in $F$."

Proposition 2.2. $\quad$ (i) Let $(X, \mathcal{S})$ be a measurable space, $Y$ a Polish space and let $\Phi: X \rightarrow F(Y)$ be a measurable function, where $F(Y)$ denotes the Effros Borel space of closed subsets of $Y$. Let $A \subseteq X \times Y$ be a measurable set (where $Y$ is equipped with its Borel $\sigma$-algebra) and $U \subseteq Y$ an open set. Then the set

$$
\left\{x \in X: U \cap \Phi(x)=\emptyset \text { or } A_{x} \cap \Phi(x) \text { is non-meager in } U \cap \Phi(x)\right\}
$$
is measurable.

(ii) Let $X$ be a Polish space and $A \subseteq X$ be Borel. Then the set

$$
\left\{F \in F(X): \exists^{*} x \in F x \in A\right\}
$$

is Borel.

Proof. (i), The proof goes exactly as in [15, 16.1]. If we let $A_{U}$ denote the set defined in (11), one checks that

- for all $S \in \mathcal{S}, V \subseteq Y$ open

$$
\begin{aligned}
(S \times V)_{U}=\{x \in X: U & \cap \Phi(x)=\emptyset \text { or } \\
& (x \in S \text { and } U \cap V \cap \Phi(x) \neq \emptyset)\} ;
\end{aligned}
$$

- $\left(\bigcup_{n} A_{n}\right)_{U}=\bigcup_{n}\left(A_{n}\right)_{U}$

- $(\sim A)_{U}=\sim \bigcap_{U_{n} \subset U} A_{U_{n}}$, where the intersection is over all $U_{n} \subseteq U$ from a fixed countable base of $Y$.

(ii) This follows from (i).

If $x$ is a Toeplitz word and $H \leq G$ is a finite index subgroup, we let

$$
\operatorname{Per}_{H}(x)=\left\{g \in G:\left.x\right|_{H g} \text { is constant }\right\} .
$$

The fact that $x$ is Toeplitz translates to $\bigcup_{H} \operatorname{Per}_{H}(x)=G$. $H$ is called an essential group of periods for $x$ if for all $g \notin H, \operatorname{Per}_{H}(x) \nsubseteq \operatorname{Per}_{H}(g \cdot x)$.

The maximal equicontinuous factor (m.e.f. for short) of a topological dynamical system $G \curvearrowright X$ is the factor generated by all equicontinuous factors of $X$. For a Toeplitz subshift $S$, this is always of the form $G \curvearrowright \lim G / H_{n}$, where $\left\{H_{n}\right\}$ is a 
decreasing sequence of essential groups of periods (see [3, Proposition 7]). Note that every such system can also be written as $G \curvearrowright \widehat{G} / K$, where $K=\bigcap_{n} \overline{H_{n}}$ with the closures taken in $\widehat{G}$. We have the following folklore lemma.

Lemma 2.3. Let $S$ be a Toeplitz subshift. Then the set of Toeplitz words in $S$ is dense $G_{\delta}$.

Proof. Let $Y$ be the m.e.f. of $S$ and $\pi: S \rightarrow Y$ be the factor map. By [3, Theorem 2], the set of Toeplitz words in $S$ can be written as

$$
\left\{x \in S: \pi^{-1}(\{\pi(x)\})=\{x\}\right\} .
$$

This can be written as $\pi^{-1}(A)$, where

$$
A=\bigcap_{\epsilon>0} \bigcup\left\{U \subseteq Y \text { open : } \operatorname{diam}\left(\pi^{-1}(U)\right)<\epsilon\right\}
$$

and this is clearly $G_{\delta}$. It is also dense by the definition of a Toeplitz subshift.

Denote

$$
\text { Тӧр }(G)=\{S \in S(G): S \text { is a Toeplitz subshift }\} .
$$

Lemma 2.3 allows us to build the following selector map that will be used throughout the paper.

Proposition 2.4. Let $G$ be a residually finite group. Then there exists a Borel map $\tau: \operatorname{Töp}(G) \rightarrow 2^{G}$ such that for all $S \in \operatorname{Töp}(G), \tau(S) \in S$ and $\tau(S)$ is a Toeplitz word.

Proof. This follows from Lemma 2.3, Proposition 2.2, and [15, 18.6].

Now we can easily deduce the following.

Lemma 2.5. Let $G$ be a residually finite group such that $\widehat{G}$ is metrizable. The map $\operatorname{Töp}(G) \rightarrow F(\widehat{G})$ which associates to a Toeplitz subshift $S$ (the conjugacy class of) the subgroup $K \leq \widehat{G}$ such that the m.e.f. of $S$ is isomorphic to $\widehat{G} / K$ is Borel.

Proof. By Proposition 2.4 for a given Toeplitz subshift $S$, we can choose in a Borel way a Toeplitz word $x \in S$. By [3], we can choose in a Borel way a sequence $L_{n}(x)$ of finite index subgroups of $G$, which are essential periods of $x$ and such that, posing $K=\bigcap_{n} \overline{L_{n}}$, we have that the m.e.f. of $S$ is isomorphic to $\widehat{G} / K$. Now it is easy to check that the map that associates to the sequence $\left\{L_{n}\right\}_{n}$ the intersection $\bigcap_{n} \overline{L_{n}}$ is Borel.

Proposition 2.6. Let $G$ be a residually finite group, $\widehat{G}$ the profinite completion of $G$ and $K \leq \widehat{G}$ a closed subgroup. Then the set Töp $(G)$ is Borel and if $\widehat{G}$ is metrizable,

$$
\operatorname{Töp}(G, K)=\left\{S \in K\left(2^{G}\right): S \text { is a Toeplitz subshift with m.e.f. } \widehat{G} / K\right\}
$$

is also Borel.

Proof. We have

$$
S \in \operatorname{Töp}(G) \Longleftrightarrow \exists^{*} x \in S x \text { is Toeplitz }
$$

and applying Proposition 2.2 yields that Töp $(G)$ is a Borel set.

The second statement follows from Lemma 2.5 


\section{3. $\mathbb{Z}$-SUBSHIFTS}

In this section, we consider $\mathbb{Z}$-subshifts and prove Theorem 1.1

Let $x \in 2^{\mathbb{Z}}$ be a Toeplitz word and $p \in \mathbb{N}$. Denote by $\operatorname{Per}_{p}(x)$ the subset of $\mathbb{Z} / p \mathbb{Z}$ defined as follows:

$$
\operatorname{Per}_{p}(x)=\{i+p \mathbb{Z}: x(i+k p)=x(i) \text { for all } k \in \mathbb{Z}\} .
$$

Let $H_{p}(x)=(\mathbb{Z} / p \mathbb{Z}) \backslash \operatorname{Per}_{p}(x)$. The elements of $H_{p}(x)$ are called $p$-holes for $x$.

We say that $x$ has separated holes 8 ] if

$$
\lim _{p \rightarrow \infty} \min \left\{|i-j|: i, j \in H_{p}(x), i \neq j\right\}=\infty .
$$

Having separated holes is a property of the subshift that does not depend on the choice of the word $x$. Thus, by the arguments in Section 2 , the condition of having separated holes defines a Borel subset of the set of all subshifts. All shifts with separated holes are regular and have topological entropy 0.

Next we recall the definition of an amenable equivalence relation. Let $E$ be a countable equivalence relation on the standard Borel space $X$. If $x \in X$ and $f: E \rightarrow \mathbb{R}$ is a function, denote by $f_{x}$ the function $[x]_{E} \rightarrow \mathbb{R}$ defined by $f_{x}(y)=$ $f(x, y) . E$ is called (1-)amenable if there exist positive Borel functions $\lambda^{n}: E \rightarrow \mathbb{R}$ such that

- $\lambda_{x}^{n} \in \ell^{1}\left([x]_{E}\right),\left\|\lambda_{x}^{n}\right\|_{1}=1$;

- $\lim _{n \rightarrow \infty}\left\|\lambda_{x}^{n}-\lambda_{y}^{n}\right\|_{1}=0$ for all $(x, y) \in E$.

By a theorem of Connes, Feldman and Weiss [2] (see also [16]), if $\mu$ is any probability measure on $X$ and $E$ is amenable, then it is hyperfinite $\mu$-almost everywhere. It is an open question whether every amenable equivalence relation is hyperfinite. See 13 for more details on amenable equivalence relations in the Borel setting.

The goal of this section is to prove the following theorem.

Theorem 3.1. The equivalence relation of isomorphism of Toeplitz $\mathbb{Z}$-subshifts with separated holes is amenable.

Proof. Let $p \in \mathbb{N}$. Denote by $\operatorname{Sym}\left(2^{p}\right)$ the set of all bijections $2^{p} \rightarrow 2^{p}$. For $\pi \in \operatorname{Sym}\left(2^{p}\right)$, let $\hat{\pi}: 2^{\mathbb{Z}} \rightarrow 2^{\mathbb{Z}}$ be defined by $\left.\hat{\pi}(x)\right|_{[k p,(k+1) p)]}=\pi\left(\left.x\right|_{[k p,(k+1) p)}\right)$ for all $k \in \mathbb{Z}$. Then if $S \subseteq 2^{\mathbb{Z}}$ is a closed $p \mathbb{Z}$-invariant set, $\hat{\pi}(S)$ is also a closed $p \mathbb{Z}$-invariant set and $\hat{\pi}$ defines an isomorphism between them (as $p \mathbb{Z}$-systems).

If $x \in 2^{\mathbb{Z}}$ and $i \in \mathbb{Z}$, denote by $x+i$ the shift of $x$ by $i$. If $S \subseteq 2^{\mathbb{Z}}$ is a Toeplitz subshift, $x \in S$ is a Toeplitz word and $p$ is a period, define $A_{p, i}(x) \subseteq S(\mathbb{Z})$ and $B^{p}: E \rightarrow \mathbb{R}$ by

$$
\begin{aligned}
A_{p, i}(x) & =\left\{\overline{\mathbb{Z} \cdot \hat{\pi}(x+i)}: \pi \in \operatorname{Sym}\left(2^{p}\right)\right\}, \\
B^{p}(S, T) & =\sum_{i<p} \chi_{A_{p, i}(x)}(T),
\end{aligned}
$$

where $\chi_{A}$ denotes the characteristic function of $A$. Note that the value of $B^{p}(S, T)$ does not depend on the choice of a Toeplitz $x \in S$. Indeed, let $x_{1}, x_{2} \in S$ be Toeplitz. We have that $S=\bigcup_{j<p} \overline{p \mathbb{Z} \cdot\left(x_{1}+j\right)}$ and there exists $j_{0}$ such that $x_{2} \in \overline{p \mathbb{Z} \cdot\left(x_{1}+j_{0}\right)}$ and therefore $\left(\right.$ as $\overline{p \mathbb{Z} \cdot\left(x_{1}+j_{0}\right)}$ is Toeplitz and thus, minimal), $\overline{p \mathbb{Z} \cdot x_{2}}=\overline{p \mathbb{Z} \cdot\left(x_{1}+j_{0}\right)}$. Then $\overline{p \mathbb{Z} \cdot \hat{\pi}\left(x_{2}+i\right)}=\overline{p \mathbb{Z} \cdot \hat{\pi}\left(x_{1}+j_{0}+i\right)}$, whence, by minimality, $\overline{\mathbb{Z} \cdot \hat{\pi}\left(x_{2}+i\right)}=\overline{\mathbb{Z} \cdot \hat{\pi}\left(x_{1}+j_{0}+i\right)}$ for every $i$ and every $\pi$. Note finally that, as we can choose $x=\tau(S)$ as per Proposition 2.4, the function $B^{p}$ is Borel.

Denote by $E$ the equivalence relation of isomorphism on Toeplitz subshifts and define $\lambda^{p}: E \rightarrow \mathbb{R}$ by

$$
\lambda^{p}(S, T)=\frac{B^{p}(S, T)}{\sum_{T^{\prime} E S} B^{p}\left(S, T^{\prime}\right)}=\frac{B^{p}(S, T)}{\left\|B_{S}^{p}\right\|_{1}} .
$$


The functions $\lambda^{p}$ clearly satisfy the first condition in the definition of amenability; in what follows, we check that if we restrict to the subshifts with separated holes, they also satisfy the second.

Recall that if $S$ and $T$ are subshifts and $f: S \rightarrow T$ is an isomorphism, then it is given by a block code, i.e., there exists $r \in \mathbb{N}$ and a function $\phi: 2^{2 r+1} \rightarrow 2$ such that for all $x \in S$,

$$
f(x)(i)=\phi\left(\left.x\right|_{[i-r, i+r]}\right) .
$$

The number $r$ is called the radius of the block code.

Let $S$ and $T$ be two Toeplitz subshifts with separated holes and let $f: S \rightarrow T$ be an isomorphism such that $f$ and $f^{-1}$ are given by block codes of radius $r$. Let $\epsilon>0$ be given. If $C \subseteq \mathbb{Z}$, let

$$
(C)_{r}=\{c+j \in \mathbb{Z} / p \mathbb{Z}: c \in C,|j| \leq r\}
$$

(Here and below, we suppress the natural quotient map $\mathbb{Z} \rightarrow \mathbb{Z} / p \mathbb{Z}$ from the notation.) Note that $\left|(C)_{r}\right| \leq(2 r+1)|C|$.

Let $x \in S$ be a Toeplitz word and let $y=f(x)$. Let $p$ be a period so big that the distance between two consecutive holes in $H_{p}(x)$ and in $H_{p}(y)$ is larger than $M=(2 r+1) / \epsilon$.

Claim 1. For all $i \notin\left(H_{p}(x)\right)_{r}$, we have $A_{p, i}(x)=A_{p, i}(y)$.

Proof. This follows from the fact that for $i \notin\left(H_{p}(x)\right)_{r}$, there exists $\pi \in \operatorname{Sym}\left(2^{p}\right)$ such that $\hat{\pi}(x+i)=y+i$. Indeed, as $y=f(x)$ and $f$ is given by a block code of radius $r,\left.y\right|_{[i+k p, i+(k+1) p)}$ only depends on $\left.x\right|_{[i+k p-r, i+(k+1) p+r)}$ and

$$
\begin{aligned}
\left.x\right|_{[i+k p-r, i+k p)]} & =\left.x\right|_{[i+(k+1) p-r, i+(k+1) p)} \\
\left.x\right|_{[i+(k+1) p, i+(k+1) p+r)} & =\left.x\right|_{[i+k p, i+k p+r)}
\end{aligned}
$$

because $(i-r, i+r) \subseteq \operatorname{Per}_{p}(x)$. Thus $\left.y\right|_{[i+k p, i+(k+1) p)}$ can be calculated from $\left.x\right|_{[i+k p, i+(k+1) p)}$ in a way independent of $k$ which gives the claim.

Claim 2. For all $i \notin H_{p}(x)$, we have $A_{p, i}(x)=A_{p, i+1}(x)$.

Proof. Indeed, let $\sigma: 2^{\mathbb{Z} / p \mathbb{Z}} \rightarrow 2^{\mathbb{Z} / p \mathbb{Z}}$ be defined by $\sigma(z)(j)=z(j-1)$, and observe that as $i \in \operatorname{Per}_{p}(x), \hat{\sigma}(x+i)=x+i+1$. Then

$$
\begin{aligned}
A_{p, i}(x) & =\left\{\overline{\mathbb{Z} \cdot \hat{\pi}(x+i)}: \pi \in \operatorname{Sym}\left(2^{p}\right)\right\} \\
& =\left\{\overline{\mathbb{Z} \cdot(\widehat{\pi \sigma})(x+i)}: \pi \in \operatorname{Sym}\left(2^{p}\right)\right\} \\
& =A_{p, i+1}(x) .
\end{aligned}
$$

Let $h_{0}, h_{1}, h_{2}, \ldots h_{J}$ enumerate the holes in $H_{p}(x)$ in circular order (so that $J+1=0)$. By the choice of $p,\left|h_{j}-h_{j+1}\right| \geq M$ for all $j$. If $f$ is a real-valued function, denote by $f^{+}$the function $\max (f, 0)$ and note that $\|f\|_{1}=\left\|f^{+}\right\|_{1}+\left\|(-f)^{+}\right\|_{1}$. We 
calculate:

$$
\begin{aligned}
\left\|\left(B_{S}^{p}-B_{T}^{p}\right)^{+}\right\|_{1} & =\left\|\left(\sum_{i<p} \chi_{A_{p, i}(x)}-\sum_{i<p} \chi_{A_{p, i}(y)}\right)^{+}\right\|_{1} \\
& \leq\left\|\sum_{j<J} \sum_{i=h_{j}-r}^{h_{j}+r} \chi_{A_{p, i}(x)}\right\|_{1} \\
& \leq\left\|\sum_{j<J}\left(\sum_{i=h_{j}+1}^{h_{j}+r} \chi_{A_{p, i}(x)}+\sum_{i=h_{j+1}-r}^{h_{j+1}} \chi_{A_{p, i}(x)}\right)\right\|_{1} \\
& \leq \sum_{j<J}((2 r+1) / M) \sum_{i=h_{j}+1}^{h_{j+1}}\left|A_{p, i}(x)\right| \\
& =((2 r+1) / M)\left\|B_{S}^{p}\right\|_{1} \\
& \leq \epsilon\left\|B_{S}^{p}\right\|_{1} .
\end{aligned}
$$

For the inequality on the second line, we use Claim 1 , and for the one on the fourth line, we use Claim 2 Similarly, $\left\|\left(B_{T}^{p}-B_{S}^{p}\right)^{+}\right\|_{1} \leq \epsilon\left\|B_{T}^{p}\right\|_{1}$, whence

$$
\left\|B_{S}^{p}-B_{T}^{p}\right\|_{1} \leq \epsilon\left(\left\|B_{S}^{p}\right\|_{1}+\left\|B_{T}^{p}\right\|_{1}\right) \text {. }
$$

Using these estimates, it is now easy to see that if $\left\{p_{n}\right\}$ is a sequence of periods such that $p_{n} \mid p_{n+1}$ for all $n$, then $\left\|\lambda_{x}^{p_{n}}-\lambda_{y}^{p_{n}}\right\|_{1} \rightarrow 0$ as $n \rightarrow \infty$.

\section{The NON-AMEnABle CASE}

Now we return to the general situation of Section 2, where $G$ is a residually finite group. We also fix a decreasing sequence $\left\{H_{n}\right\}$ of finite index, normal subgroups with trivial intersection, to be determined later. We also denote by $\hat{G}$ the inverse $\operatorname{limit} \lim G / H_{n}$ (which is a group quotient of the profinite completion $\widehat{G}$ ) and by $\pi_{n}: \hat{G \rightarrow G} / H_{n}$ the natural projections.

The goal of this section is to prove Theorem 1.2. The proof proceeds by constructing a probability measure on the set of Toeplitz subshifts which is invariant under an appropriate action of $G$ contained in the isomorphism relation. We then check that the point stabilizers for this action are amenable and conclude that if $G$ is non-amenable, then the isomorphism equivalence relation is not amenable either.

4.1. The left and right actions. First, we describe the construction of the measure. Let $A_{n}=\left(H_{n-1} / H_{n}\right) \backslash\left\{H_{n}\right\}$ and let $Z=2^{\sqcup_{n}} A_{n}$. Let $Y=\{y \in \hat{G}: y \notin G\}$. Consider the maps

$$
Y \times Z \stackrel{\sigma}{\longrightarrow} 2^{G} \stackrel{\rho}{\longrightarrow} S(G)
$$

defined as follows. If $(y, z) \in Y \times Z$, define $\sigma(y, z)$ by

$$
\sigma(y, z)(h)=z\left(\pi_{n_{0}}\left(y^{-1} h\right)\right), \quad \text { where } n_{0}=\min \left\{n: \pi_{n}(y) \neq \pi_{n}(h)\right\} .
$$

Define $\rho: 2^{G} \rightarrow S(G)$ by $\rho(x)=\overline{G \cdot x}$. Let $\theta=\rho \circ \sigma$.

$G$ acts on $2^{G}$ on both sides: on the left, by

$$
(g \cdot x)(h)=x\left(g^{-1} h\right)
$$

and on the right, by

$$
(x \cdot g)(h)=x\left(h g^{-1}\right)
$$

and the two actions commute. Note that if $S \subseteq 2^{G}$ is a subshift and $g \in G$, then $S \cdot g$ is a subshift too, so we have a right action $S(G) \curvearrowleft G$. Moreover, for every fixed $g \in G$, the map

$$
S \rightarrow S \cdot g, \quad x \mapsto x \cdot g
$$

is an isomorphism of subshifts. 
The space $Y \times Z$ is also equipped with commuting left and right actions of $G$, defined as follows. For $y \in Y$ and $g \in G$, define $g \cdot y=g y$ and $y \cdot g=y g$ (recall that $G \subseteq \hat{G})$. For $z \in Z$, define $g \cdot z=z$ and $(z \cdot g)(a)=z\left(g a g^{-1}\right)$ for all $a \in \bigsqcup_{n} A_{n}$. Equip $Y \times Z$ with the diagonal left and right actions.

Lemma 4.1. The maps $\sigma$ and $\rho$ commute with both the left and right actions of $G$ on the respective spaces.

Proof. We only check that $\sigma$ commutes with the right actions. Suppose that $\left(y_{1}, z_{1}\right),\left(y_{2}, z_{2}\right)$ are elements of $Y \times Z$ such that $\left(y_{1}, z_{1}\right) \cdot g=\left(y_{2}, z_{2}\right)$. Let $x_{1}=$ $\sigma\left(y_{1}, z_{1}\right)$ and $x_{2}=\sigma\left(y_{2}, z_{2}\right)$. Note that for every $h \in G$ we have

$$
\min \left\{n: \pi_{n}\left(y_{1}\right) \neq \pi_{n}\left(h g^{-1}\right)\right\}=\min \left\{n: \pi_{n}\left(y_{1} g\right) \neq \pi_{n}(h)\right\} .
$$

Write $n_{0}(h)$ for this common value. Thus,

$$
\begin{aligned}
\left(x_{1} \cdot g\right)(h) & =x_{1}\left(h g^{-1}\right)=z_{1}\left(\pi_{n_{0}(h)}\left(y_{1}^{-1} h g^{-1}\right)\right), \quad \text { and } \\
x_{2}(h) & =z_{2}\left(\pi_{n_{0}(h)}\left(y_{2}^{-1} h\right)\right)=z_{2}\left(\pi_{n_{0}(h)}\left(g^{-1} y_{1}^{-1} h\right)\right) .
\end{aligned}
$$

But

$$
\begin{aligned}
z_{2}\left(\pi_{n_{0}(h)}\left(g^{-1} y_{1}^{-1} h\right)\right) & \left.=z_{1}\left(g \pi_{n_{0}(h)}\left(g^{-1} y_{1}^{-1} h\right) g^{-1}\right)\right) \\
& =z_{1}\left(\pi_{n_{0}(h)}\left(y_{1}^{-1} h g^{-1}\right)\right)
\end{aligned}
$$

so $x_{1} \cdot g=x_{2}$, as needed.

We call an element $z \in Z$ proper if it takes both values 0 and 1 infinitely many times. Note that $z$ is proper if and only if for every (any) $y \in Y, G \cdot \sigma(y, z)$ is infinite.

Lemma 4.2. For any $y \in Y$ and proper $z \in Z, \sigma(y, z)$ is a Toeplitz word and the m.e.f. of $\overline{G \cdot \sigma(y, z)}$ is isomorphic to $\hat{G}$. In particular, the subshift $\overline{G \cdot \sigma(y, z)}$ is free.

Proof. Write $x=\sigma(y, z)$. First note that for any $g \in G$, the value $x(g)$ is assumed on the whole $H_{n_{0}}$-coset $\pi_{n_{0}}\left(y^{-1} g\right)$, where $n_{0}=\min \left\{n: \pi_{n}(y) \neq \pi_{n}(g)\right\}$, showing that $x$ is Toeplitz.

To calculate the m.e.f., by [3, it suffices to observe that for all $n, H_{n}$ is an essential group of periods for $x$. Indeed, using the fact that $z$ is proper, we have that $\operatorname{Per}_{H_{n}}(x)=G \backslash \pi_{n}(y)$ which is not contained in $\operatorname{Per}_{H_{n}}(g \cdot x)=G \backslash \pi_{n}(g \cdot y)$ for any $g \notin H_{n}$.

For the final claim, just note that as $\bigcap_{n} H_{n}=\left\{1_{G}\right\}$ and the $H_{n}$ are normal, the translation action $G \curvearrowright \hat{G}$ is free.

The main ingredient for the proof of the theorem is the following lemma.

Lemma 4.3. For all proper $z_{1}, z_{2} \in Z$ and all $y_{1}, y_{2} \in Y$,

$$
z_{1} \neq z_{2} \Longrightarrow \theta\left(y_{1}, z_{1}\right) \neq \theta\left(y_{2}, z_{2}\right) \text {. }
$$

Proof. Let $x_{i}=\sigma\left(y_{i}, z_{i}\right)$. To show that $\overline{G \cdot x_{1}} \neq \overline{G \cdot x_{2}}$, it suffices to find a "subword" of $x_{2}$ that does not occur in $x_{1}$, i.e., a finite set $F \subseteq G$ such that

$$
\forall g \in G \exists f \in F \quad x_{1}(g f) \neq x_{2}(f) .
$$

Let $a_{0} \in A_{n}$ be such that $z_{1}\left(a_{0}\right) \neq z_{2}\left(a_{0}\right)$. By replacing $\left(y_{1}, z_{1}\right)$ and $\left(y_{2}, z_{2}\right)$ with $g_{1} \cdot\left(y_{1}, z_{1}\right)$ and $g_{2} \cdot\left(y_{2}, z_{2}\right)$ (which does not change either $z_{i}$ or $\theta\left(y_{i}, z_{i}\right)$ ) for suitably chosen $g_{1}, g_{2} \in G$, we may assume that $\pi_{n}\left(y_{1}\right)=\pi_{n}\left(y_{2}\right)=1_{G / H_{n}}$. Let $f_{0}, f_{1} \in G$ be such that $\pi_{n}\left(f_{0}\right)=\pi_{n}\left(f_{1}\right), x_{2}\left(f_{0}\right)=0, x_{2}\left(f_{1}\right)=1$ (such $f_{0}, f_{1}$ exist by the assumption that $z_{2}$ is proper). Let $f \in G$ be such that $\pi_{n}(f)=a_{0}$ and finally, let $F=\left\{f, f_{0}, f_{1}\right\}$. 
Next we show that this $F$ works. Let $g \in G$ be arbitrary. We distinguish two cases: $g \in H_{n}$ and $g \notin H_{n}$. Let first $g \in H_{n}$. We check that $x_{1}(g f) \neq x_{2}(f)$. Indeed,

$$
x_{1}(g f)=z_{1}\left(\pi_{n}\left(y_{1}^{-1} g f\right)\right)=z_{1}\left(\pi_{n}(f)\right)=z_{1}\left(a_{0}\right)
$$

and

$$
x_{2}(f)=z_{2}\left(\pi_{n}\left(y_{2}^{-1} f\right)\right)=z_{2}\left(\pi_{n}(f)\right)=z_{2}\left(a_{0}\right),
$$

which are different by the choice of $a_{0}$.

Suppose now that $g \notin H_{n}$. We will show that $x_{1}\left(g f_{0}\right)=x_{1}\left(g f_{1}\right)$ which will complete the proof (as $x_{2}\left(f_{0}\right) \neq x_{2}\left(f_{1}\right)$ ). Indeed, observe that the least $k$ for which $\pi_{k}\left(g f_{i}\right) \neq 1_{G / H_{k}}$ is at most $n$ and is the same for $i=0,1\left(\right.$ as $\left.\pi_{n}\left(f_{0}\right)=\pi_{n}\left(f_{1}\right)\right)$. Recall also that $\pi_{k}\left(y_{1}\right)=\pi_{k}\left(y_{2}\right)=1_{G / H_{k}}$. Now we have

$$
\begin{aligned}
x_{1}\left(g f_{0}\right) & =z_{1}\left(\pi_{k}\left(y_{1}^{-1} g f_{0}\right)\right)=z_{1}\left(\pi_{k}\left(g f_{0}\right)\right) \\
& =z_{1}\left(\pi_{k}\left(g f_{1}\right)\right)=x_{1}\left(g f_{1}\right),
\end{aligned}
$$

completing the proof.

4.2. A.e. amenable stabilizers. Let $\lambda$ be the Haar measure on $\hat{G}$ and note that as $\lambda(Y)=1$, we can consider $\lambda$ as a measure on $Y$. Let $\nu$ be the Bernoulli $\left(\frac{1}{2}, \frac{1}{2}\right)$ measure on $Z=2^{\bigsqcup_{n}} A_{n}$. Equip $Y \times Z$ with the product measure $\lambda \times \nu$. It is clear that this measure is invariant under both the left and the right action of $G$ (but for us it will be the right one that will be important). Let $\mu=\theta_{*}(\lambda \times \nu)$.

Lemma 4.4. The measure $\mu$ concentrates on the set of free, Toeplitz G-subshifts and is invariant under the right action.

Proof. This follows from Lemma 4.2 and the fact that $\nu$ concentrates on the set of proper elements of $Z$. Invariance follows directly from Lemma 4.1]

For $g \in G$, denote by $C(g)$ the centralizer of $g$ in $G$, i.e., the set of all elements of $G$ that commute with $g$. Let

$$
Z_{f}(G)=\{g \in G: C(g) \text { has finite index in } G\} .
$$

Lemma 4.5. $Z_{f}(G)$ is an amenable, normal subgroup of $G$.

Proof. It is clear that $Z_{f}(G)$ is a normal subgroup of $G$. To see that it is amenable, note that the centralizers of all elements in $Z_{f}(G)$ have finite index in $Z_{f}(G)$. Hence, $Z_{f}(G)$ has finite conjugacy classes and this implies amenability (Leptin [18]).

Next we choose a suitable sequence $\left\{H_{n}\right\}$ of finite index, normal subgroups of $G$ that will allow us to prove the theorem. Note that the residual finiteness of $G$ implies that for any finite index subgroup $H \triangleleft G$ and $g \in G \backslash Z_{f}(G)$ there exists a finite index subgroup $H^{\prime} \leq H$ normal in $G$ such that for some $C \in H / H^{\prime}$ we have $g C g^{-1} \neq C$. Enumerate $G \backslash Z_{f}(G)$ as $\left\{g_{n}: n \in \mathbb{N}\right\}$ and construct inductively $\left\{H_{n}: n \in \mathbb{N}\right\}$ a decreasing sequence with trivial intersection such that

(2) for all $m \geq n$ there exists $C \in H_{m} / H_{m+1}$ such that $g_{n}^{-1} C g_{n} \neq C$.

Proof of Theorem 1.2. We will show that the right action of $G$ on $\operatorname{Töp}(G)$ has $\mu$-a.e amenable stabilizers. Recall that (see, e.g., [11, Lemma 3.6]) if a measurepreserving action of a group has amenable stabilizers and induces a hyperfinite equivalence relation, then the group is amenable. Thus, if $G$ is non-amenable, we obtain that the orbit equivalence relation induced by the right action of $G$ on the set of Toeplitz $G$-subshifts is not hyperfinite and, as it is contained in the topological conjugacy relation, the latter is not hyperfinite either. 
By Lemma 4.5, it is enough to show that there is a measure 1 subset $A$ of Töp $(G)$ such that the stabilizer of every element in $A$ is contained in $Z_{f}(G)$. Since $G$ is countable, it suffices to see that for every $g \in G \backslash Z_{f}(G)$, the set

$$
\{(y, z) \in Y \times Z: \theta(y, z) \cdot g \neq \theta(y, z)\}
$$

has measure 1. By Lemma 4.3, it is enough to show that $\{z \in Z: z \cdot g \neq z\}$ is of measure 1. As $g \notin Z_{f}(G)$, by (2), for almost all $n$, there is an element, say $C_{n} \in H_{n+1} / H_{n}$ such that $g C_{n} g^{-1} \neq C_{n}$. By the definition of the action of $G$ on $Z$, for any $z \in Z$ such that there exists $n$ with $z\left(C_{n}\right) \neq z\left(g C_{n} g^{-1}\right)$, we have $z \neq z \cdot g$. But the latter condition is clearly satisfied on a measure 1 set. This completes the proof of the theorem.

Remark 4.6. Note that in case $G$ is a non-cyclic free group, the right action of $G$ on Töp $(G)$ is free $\mu$-a.e. because $Z_{f}(G)$ is trivial (centralizers of non-trivial elements of free groups are cyclic). This implies in particular that the equivalence relation of topological conjugacy of Toeplitz $G$-subshifts embeds a free, measure-preserving action of a free group.

\section{The GROUPOID VIEWPOINT}

The equivalence relation of isomorphism of subshifts is naturally given by an action of a groupoid rather than a group. Recall that a groupoid is a small category where each arrow is invertible. We will only be interested in countable Borel groupoids, defined as follows (see also [20]). A countable Borel groupoid $\Gamma$ is a tuple $(X, A, s, r, \circ)$, where $X$ is a standard Borel space of objects, $A$ is a standard Borel space of arrows, $s: A \rightarrow X$ is a Borel map specifying the source of each arrow and $r: A \rightarrow X$ a Borel map specifying its range. $\circ$ is a partial Borel map $A \times A \rightarrow A$ which represents the composition of arrows; $f \circ g$ is defined whenever $r(g)=s(f)$. Of course, we require that $(X, A, \circ)$ be a groupoid. Furthermore, we require that $r^{-1}(\{x\})$ and $s^{-1}(\{x\})$ be countable sets for every $x \in X$. By the Lusin-Novikov selection theorem, there exist sequences of Borel maps $P_{n}: X \rightarrow A$ and $Q_{n}: X \rightarrow A$ such that $P_{n}(x)$ enumerate $s^{-1}(x)$ and $Q_{n}(x)$ enumerate $r^{-1}(x)$ for every $x$. If $x, y \in X$, we will denote by $A(x, y)$ the set of arrows between $x$ and $y$. We will sometimes identify a groupoid with the set of its arrows as the other information can be recovered from it.

Every countable Borel groupoid $\Gamma$ gives rise to a countable Borel equivalence relation $E_{\Gamma}$ on $X$ defined by

$$
x E_{\Gamma} y \Longleftrightarrow \exists f \in A s(f)=x \text { and } r(f)=y .
$$

Conversely, every countable equivalence relation can be viewed as groupoid, where there is a unique arrow between every pair of equivalent elements of $X$.

Now let $G$ be a residually finite group as before, $\left\{H_{n}\right\}$ be a sequence of finite index, normal subgroups of $G$, and let $X$ be the standard Borel space of of all Toeplitz subshifts of $G$ with m.e.f. $\hat{G}=\lim G / H_{n}$. Let $A$ be the set of all isomorphisms between elements of $X$. Recalling that every such isomorphism is given by a block code (which is a finite object), it is easy to endow $A$ with a standard Borel structure so that $(X, A, \circ)$ becomes a countable Borel groupoid (o is just composition of maps). The equivalence relation generated by this groupoid is exactly isomorphism of subshifts. (All of this is defined for arbitrary subshifts of countable groups; however, below we will need that they be Toeplitz.)

Note that for every $S \in X$ and every $x \in S$, there exists a unique factor map $\pi_{x}: S \rightarrow \hat{G}$ such that $\pi(x)=1_{\hat{G}}$. (Existence follows from the fact that if $\pi: S \rightarrow \hat{G}$ is any $G$-map, then post-composing $\pi$ with right multiplication by $\pi(x)^{-1}$ yields a map that sends $x$ to $1_{\hat{G}}$.) Let $\tau: X \rightarrow 2^{G}$ be a Borel map that selects for every 
$S \in X$, a Toeplitz word $\tau(S) \in S$ as given by Proposition 2.4. Now we can define a Borel cocycle $\alpha_{0}: \Gamma \rightarrow \hat{G}$ by

$$
\alpha_{0}(f)=\pi_{\tau(r(f))}(f(\tau(s(f))))^{-1} .
$$

Recall that a cocycle is just a map $\Gamma \rightarrow \hat{G}$ that satisfies $\alpha(f \circ g)=\alpha(f) \alpha(g)$. Two cocycles $\alpha, \beta$ are cohomologous if there exists a Borel map $F: X \rightarrow \hat{G}$ such that $\beta(f)=F(r(f))^{-1} \alpha(f) F(s(f))$ for all $f \in \Gamma$. Note that changing the selection map $\tau$ transforms $\alpha_{0}$ into a cohomologous cocycle. We also have a natural Borel homomorphism $\rho: \Gamma \rightarrow E$ defined by

$$
\rho(f)=(s(f), r(f)) .
$$

Now we specialize to the case $G=\mathbb{Z}$. We have the following proposition, which basically follows from a result by Downarowicz, Kwiatkowski, and Lacroix 6].

Proposition 5.1. Let $\Delta=\operatorname{ker} \alpha_{0}=\left\{f \in \Gamma: \alpha_{0}(f)=0\right\}$. Then the equivalence relation $E_{\Delta}$ is hyperfinite.

Proof. For a period $p$, define the finite equivalence relation $E_{p}$ on $\operatorname{Töp}(\mathbb{Z})$ by

$$
S E_{p} T \Longleftrightarrow \exists \sigma \in \operatorname{Sym}\left(2^{p}\right) \hat{\sigma}(\tau(S))=\tau(T) .
$$

It follows from [6. Theorem 1] that

$$
\begin{aligned}
E_{\Delta} & =\{(S, T): \exists f: S \rightarrow T \text { isomorphism such that } f(\tau(S))=\tau(T)\} \\
& =\bigcup_{p} E_{p},
\end{aligned}
$$

showing that $E_{\Delta}$ is hyperfinite.

If $S$ is a subshift, the centralizer $C(S)$ of $S$ is the group of automorphisms of $S$, or equivalently, the group of arrows from $S$ to itself. Note that by our observations above, if $S \in X$, then $C(S)$ embeds in $\hat{G}$ and is therefore an abelian group. So, in some sense, the grooupoid $\Gamma$ differs from the equivalence relation $E_{\Gamma}$ only a little.

We finally observe that the existence of the cocycle $\alpha_{0}$ gives some restrictions on the groupoid $\Gamma$. For example, using Popa's cocycle superrigidity results [22], it is easy to prove that $\Gamma$ does not embed the groupoid of the free part of any Bernoulli action of an infinite property (T) group. However, it is not clear how to conclude anything from that about the equivalence relation $E$; in particular, we do not know whether $E$ is universal.

\section{REFERENCES}

[1] John D. Clemens. Isomorphism of subshifts is a universal countable Borel equivalence relation. Israel J. Math., 170:113-123, 2009.

[2] Alain Connes, Jacob Feldman, and Benjamin Weiss. An amenable equivalence relation is generated by a single transformation. Ergodic Theory Dynamical Systems, 1(4):431-450 (1982), 1981.

[3] María Isabel Cortez and Samuel Petite. $G$-odometers and their almost one-to-one extensions. J. Lond. Math. Soc. (2), 78(1):1-20, 2008.

[4] María Isabel Cortez and Samuel Petite. Invariant measures and orbit equivalence for generalized Toeplitz subshifts. Groups Geom. Dyn., 8(4):1007-1045, 2014.

[5] R. Dougherty, S. Jackson, and A. S. Kechris. The structure of hyperfinite Borel equivalence relations. Trans. Amer. Math. Soc., 341(1):193-225, 1994.

[6] T. Downarowicz, J. Kwiatkowski, and Y. Lacroix. A criterion for Toeplitz flows to be topologically isomorphic and applications. Colloq. Math., 68(2):219-228, 1995.

[7] Tomasz Downarowicz. The Choquet simplex of invariant measures for minimal flows. Israel J. Math., 74(2-3):241-256, 1991.

[8] Tomasz Downarowicz. Survey of odometers and Toeplitz flows. In Algebraic and topological dynamics, volume 385 of Contemp. Math., pages 7-37. Amer. Math. Soc., Providence, RI, 2005. 
[9] S. Gao, S. Jackson, and B. Seward. Group colorings and Bernoulli subflows. Memoirs of the American Mathematical Society. to appear.

[10] Thierry Giordano, Ian F. Putnam, and Christian F. Skau. Full groups of Cantor minimal systems. Israel J. Math., 111:285-320, 1999.

[11] Greg Hjorth. Non-treeability for product group actions. Israel J. Math., 163:383-409, 2008.

[12] Greg Hjorth and Alexander S. Kechris. Rigidity theorems for actions of product groups and countable Borel equivalence relations. Mem. Amer. Math. Soc., 177(833):viii+109, 2005.

[13] S. Jackson, A. S. Kechris, and A. Louveau. Countable Borel equivalence relations. J. Math. Log., 2(1):1-80, 2002.

[14] Kate Juschenko and Nicolas Monod. Cantor systems, piecewise translations and simple amenable groups. Ann. of Math. (2), 178(2):775-787, 2013.

[15] Alexander S. Kechris. Classical descriptive set theory, volume 156 of Graduate Texts in Mathematics. Springer-Verlag, New York, 1995.

[16] Alexander S. Kechris and Benjamin D. Miller. Topics in orbit equivalence, volume 1852 of Lecture Notes in Mathematics. Springer-Verlag, Berlin, 2004.

[17] Fabrice Krieger. Sous-décalages de Toeplitz sur les groupes moyennables résiduellement finis. J. Lond. Math. Soc. (2), 75(2):447-462, 2007.

[18] H. Leptin. Zur harmonischen Analyse klassenkompakter Gruppen. Invent. Math., 5:249-254, 1968.

[19] Douglas Lind and Brian Marcus. An introduction to symbolic dynamics and coding. Cambridge University Press, Cambridge, 1995.

[20] Martino Lupini. Polish groupoids and functorial complexity. 2014. preprint, arxiv.org/abs/1407.6671.

[21] Hiroki Matui. Some remarks on topological full groups of Cantor minimal systems. Internat. J. Math., 17(2):231-251, 2006.

[22] Sorin Popa. Cocycle and orbit equivalence superrigidity for malleable actions of $w$-rigid groups. Invent. Math., 170(2):243-295, 2007.

[23] Simon Thomas. Topological full groups of minimal subshifts and just-infinite groups. In Proceedings of the 12th Asian Logic Conference, 2012.

[24] Jay Williams. Isomorphism of finitely generated solvable groups is weakly universal. Journal of Pure and Applied Algebra. to appear.

Marcin Sabok, Department of Mathematics and Statistics, McGill University, 805, Sherbrooke Street West Montreal, Quebec, Canada H3A 2K6 and Institute of Mathematics, Polish Academy of Sciences, Śniadeckich 8, 00-655 Warszawa, Poland

E-mail address: marcin.sabok@mcgill.ca

Todor Tsankov, Institut de Mathématiques de Jussieu-PRG, Université Paris Diderot, 75205 PARIS CEDEX 13

E-mail address: todor@math.univ-paris-diderot.fr 